NOTE

\title{
Post-irradiation colouration of Gafchromic EBT radiochromic film
}

\author{
Tsang Cheung ${ }^{1}$, Martin J Butson ${ }^{1,2}$ and Peter K N Yu ${ }^{1}$ \\ ${ }^{1}$ Deptment of Physics and Materials Science, City University of Hong Kong, Kowloon Tong, \\ Hong Kong \\ 2 Department of Medical Physics, Illawarra Cancer Care Centre, Crown St, Wollongong, \\ NSW 2500, Australia \\ E-mail: butsonm@iahs.nsw.gov.au
}

Received 22 March 2005, in final form 5 July 2005

Published 5 October 2005

Online at stacks.iop.org/PMB/50/N281

\begin{abstract}
Gafchromic EBT (International Specialty Products, NJ, USA), radiochromic film is one of the newest radiation-induced auto-developing X-ray analysis films available for therapeutic radiation dosimetry in radiotherapy applications. Part of any radiochromic film product which undergoes a polymerization reaction for automatic darkening is an associated post-irradiation colouration whereby the film continues to darken after irradiation has ceased. The Gafchromic EBT film has been shown to produce an approximate $6 \%$ to $9 \%$ increase in postirradiation optical density within the first $12 \mathrm{~h}$ of irradiation within the $1 \mathrm{~Gy}$ to 5 Gy dose range. This is compared to approximately $13 \%, 15 \%$ and $19 \%$ for MD-55-2, XR type T and HS radiochromic film, respectively. It is also shown that the EBT film's post-irradiation growth stabilizes to within $1 \%$ within the first $6 \mathrm{~h}$. Thus EBT provides a reduced post-irradiation growth effect. However, to increase the accuracy of the film analysis, it is recommended that films be left for a significant period (at least $6 \mathrm{~h}$ ) before the analysis is performed to provide a high level of accuracy. Also, calibration films must be read out with the same post-irradiation time to further enhance the accuracy of dosimetry.
\end{abstract}

\section{Introduction}

Most radiochromic film products have active components, which include a radiation-sensitive monomer (Butson et al 2003). Upon irradiation, this active monomer polymerizes to form a polymer coloured dye; thus, the automated darkening process upon irradiation with x-rays takes place. The final colour, or more correctly the absorption spectrum, is dependent on the proprietary chemical within the film itself. McLaughlin (1996) showed that radiation-induced reactions can have an incubation period of at least $1 \mu \mathrm{s}$. He also showed that polymerization can proceed after irradiation has ceased causing a post-exposure density growth which manifests 
itself as a significant increase in the optical absorption. This fact is well known within the medical physics community through the use of various radiochromic film products and the general rule of leaving films for a period of $24 \mathrm{~h}$ before readout is performed (Ali et al 2003, 2005, Akhaven et al 2002, Murphy et al 2003). Reinstein (Reinstein et al 1998) also showed a technique using heat treatment to speed up the reaction time for post-irradiation colouration for some film types. Recently a new radiochromic film product, Gafchromic EBT film (International Specialty Products, NJ, USA), has been commercially released which produces a different absorption spectrum from older radiochromic film products. The film also produces a higher dose sensitivity which more closely matches the dose criteria for radiotherapy applications. This short note investigates the post-irradiation colouration effects of this new film and compares results to the older conventional film.

\section{Materials and methods}

Gafchromic EBT radiochromic film (batch no. K02b28XRT) was investigated for this postirradiation colouration study. For dose delivery, the films were positioned in a solid water (Constanitinou et al 1982) phantom of dimensions $30 \mathrm{~cm} \times 30 \mathrm{~cm} \times 30 \mathrm{~cm}$ (GAMMEX RMI, WI, USA). The films were irradiated using a standard $10 \mathrm{~cm} \times 10 \mathrm{~cm} 6 \mathrm{MV}$ x-ray field at a $100 \mathrm{~cm}$ source-to-surface distance at the dmax position of $1.5 \mathrm{~cm}$ depth. The irradiated films were placed perpendicular to the central axis of the beam. Precautions in handling of radiochromic film outlined by Butson et al 2003 were used. The film during storage and film analysis was kept at temperatures of $22{ }^{\circ} \mathrm{C} \pm 2{ }^{\circ} \mathrm{C}$ to reduce any effects of time- and temperature-dependent evolution or readout of the absorption spectra of the film as is the case found for the MD-55 Gafchromic film (Meigooni et al 1996). The film is only removed from a light tight envelope during irradiation and readout to reduce any effects of ambient light (Butson et al 1998). The absorption spectra results for post-irradiation colouration measurements were obtained using a Shimadzu UV-160 UV-visible recording spectrophotometer (Butson et al 2002). The wavelength range of the analysis was from $500 \mathrm{~nm}$ to $700 \mathrm{~nm}$ in $5 \mathrm{~nm}$ steps except at the absorption peaks, where the maximum was found using a step size of $1 \mathrm{~nm}$. The Shimadzu UV-160 has a spectral bandwidth of $3 \mathrm{~nm}$ with an accuracy of $\pm 0.5 \mathrm{~nm}$. The film was held in a quartz holding container. The films after initial radiation were tested immediately and at the given time intervals quoted. For measurements using the photo spectrometer, a time lag of approximately 6 min was needed from the irradiation time to bring the films to the spectrometer machine located in another section of the hospital. Measurements were also performed on the Gafchromic MD-55-2 film (batch no. H1146MD55), Gafchromic HS film (batch no. K0223HS) and Gafchromic XR type T film (batch no. K02b28XRT) for comparison. The experiments were performed ten times and the results show the average value and uncertainties for these ten experiments.

\section{Results and discussion}

Figure 1 shows the absorption spectra for the Gafchromic EBT film in the visible $500 \mathrm{~nm}-$ $700 \mathrm{~nm}$ region for a film given 2 Gy applied dose by a $6 \mathrm{MV}$ linear accelerator produced $\mathrm{x}$-ray beam over a given time period up to $24 \mathrm{~h}$. As can be seen, the absorption spectra for the film produce two pronounced peaks which are located at $636 \mathrm{~nm}$ and $585 \mathrm{~nm}$; however, a general increase in optical density was seen over the entire range of wavelengths with the largest increase obtained at the $636 \mathrm{~nm}$ peak. Figure 2 highlights the post-irradiation colouration for the EBT film when analysed at various dose levels ranging from $1 \mathrm{~Gy}$ up to 


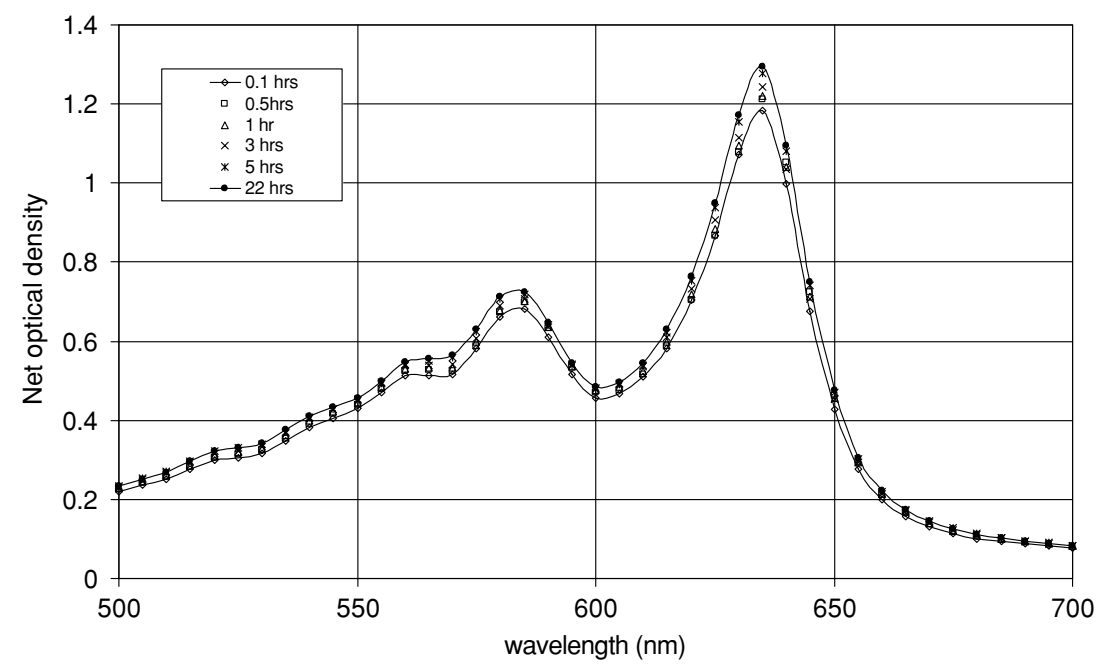

Figure 1. Absorption spectra produced post-irradiation for EBT Gafchromic film showing increase in optical density across entire measured wavelengths.

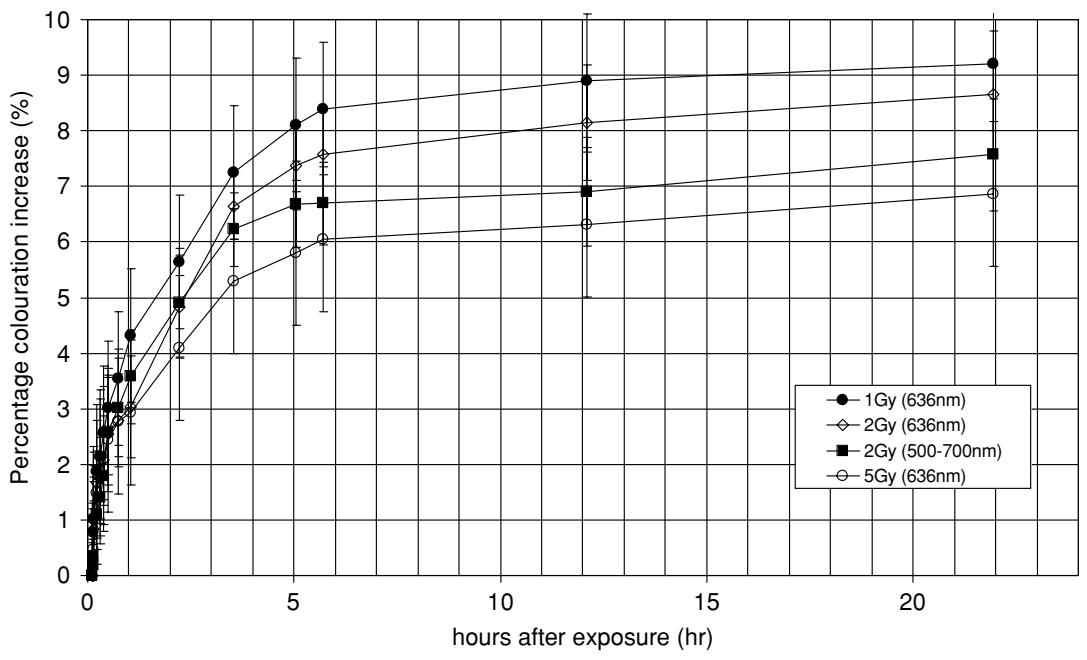

Figure 2. Percentage increase in optical density for EBT film post-irradiation at various dose levels.

5 Gy along with two specific bandwidths of analysis, these being the $636 \mathrm{~nm}$ peak wavelength and the $500 \mathrm{~nm}-700 \mathrm{~nm}$ average. Results show that an approximate 7\%-9\% increase in colouration occurs up to $24 \mathrm{~h}$ post-irradiation. In absolute OD terms the increase in postirradiation colouration is dependent on the magnitude of the delivered dose. Thus, a standard 'post-irradiation colouration' subtraction cannot be performed to remove the effects of postirradiation colouration. Approximately $1 \%$ of this colouration is from $6 \mathrm{~h}$ after irradiation. Variations and uncertainties in these values are shown on the graph and include the variations in results found from 10 film experiments to $1 \mathrm{SD}$ of the mean. The zero value was measured $0.1 \mathrm{~h}$ after irradiation $(6 \mathrm{~min})$ due to the time constraints of getting the film from the accelerator 


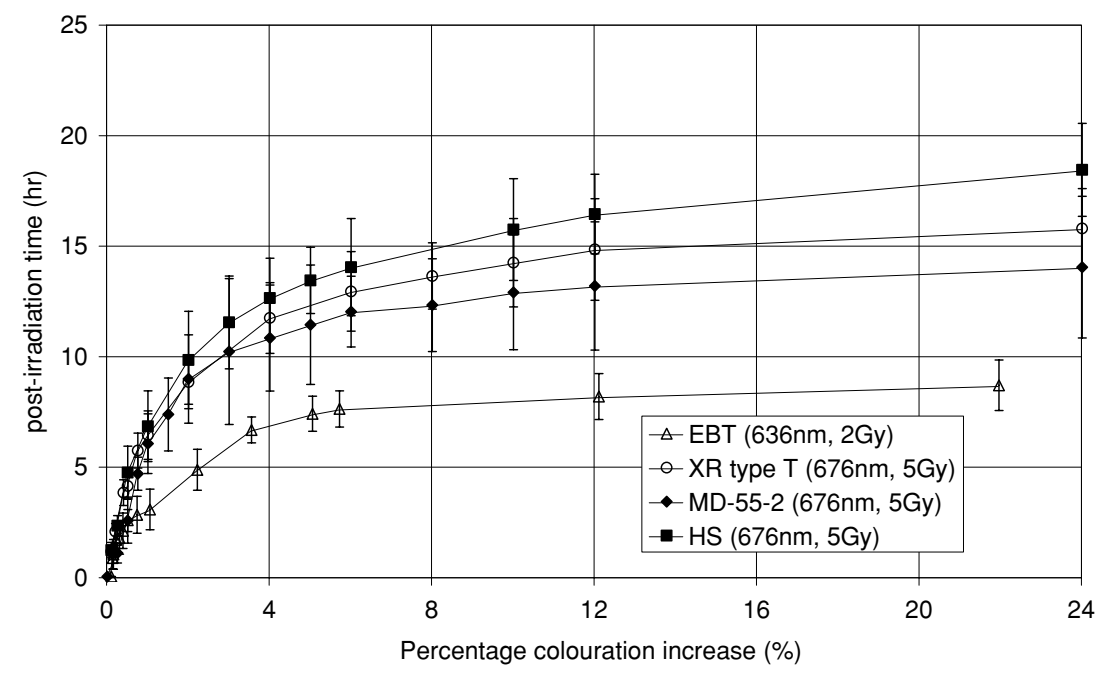

Figure 3. Comparison of post-irradiation colouration for various radiochromic film types. EBT (2 Gy applied dose), XR type T, HS and MD-55-2 (5 Gy applied dose).

to the photo-spectrometer located in another area within our department. By projecting a line of best-fit back through the post-irradiation colouration curves, we estimate that the values quoted above would increase by approximately $2 \%$ overall. This produces an extrapolated estimate of $9 \%-11 \%$ increase in colouration for the EBT film at 2 Gy incident dose. As mentioned earlier, the majority of this increase occurs within the first $6 \mathrm{~h}$ with approximately $1 \%$ variation after this time. These values for time are reduced compared to older varieties of Gafchromic film as shown in figure 3. Figure 3 also shows the reduced percentage dose increase of EBT film compared to MD-55-2, XR Type T and HS Gafchromic. Results show that 1 day after irradiation the increase in colouration is $9 \%, 14 \%, 16 \%$ and $18 \%$ for EBT, MD-55-2, XR type T and HS, respectively. Also the percentage increase from $6 \mathrm{~h}$ to $24 \mathrm{~h}$ post-irradiation was $1 \%, 2 \%, 3 \%, 4 \%$ for the same films. Thus the EBT film does undergo post-irradiation colouration; however, the magnitude is decreased compared to other products and the time for the majority of post-irradiation colouration is reduced to within $6 \mathrm{~h}$. The uncertainty of post-irradiation colouration is also smaller for EBT film. The combination of the reduced uncertainty magnitude and time frame for colouration makes EBT film more clinically accurate and useful than its predecessors. This length of time may still prove to be restrictive in some applications, so immediate results will require standard films to receive exactly the same time period of post-irradiation before readout to minimize errors relative to post-irradiation colouration.

\section{Conclusions}

Gafchromic EBT radiochromic film produces a post-irradiation colouration effect of approximately 9\%-11\% which mostly occurs within the first $6 \mathrm{~h}$. This results in a reduced quantity of post-irradiation colouration compared to older types of radiochromic film and occurs over a shorter time period. It is recommended to still leave films for a period of $6 \mathrm{~h}$ so that the majority of post-irradiation colouration can occur to improve stability of measurements; however, if immediate results are required, then the constraint of standard films having the same time period of post-irradiation must be applied. 


\section{Acknowledgments}

This work has been fully supported by a grant from the Research Grants Council of HKSAR, China (project no. CityU 100603). Thanks to Ethan Butson for assistance in film measurements.

\section{References}

Akhavan A, Sohrabpour M and Sharifzadeh M 2002 Preparation of a new chemical radiochromic film dosimeter Radiat. Phys. Chem. $63773-5$

Ali I, Costescu C, Vicic M, Dempsey J F and Williamson J F 2003 Dependence of radiochromic film optical density post-exposure kinetics on dose and dose fractionation Med. Phys. 30 1958-67

Ali I, Williamson J F, Costescu C and Dempsey J F 2005 Dependence of radiochromic film response kinetics on fractionated doses Appl. Radiat. Isot. 62 609-17

Butson M J, Cheung T and Yu P K 2002 Corresponding dose response of radiographic film with layered Gafchromic film Phys. Med. Biol. 47 N285-9

Butson M J, Yu K N, Cheung T and Metcalfe P E 2003 Radiochromic film for Medical Radiation Dosimetry, Mater. Sci. Eng. R 41 61-120

Butson M, Yu P and Metcalfe P 1998 Effects of readout light sources and ambient light on radiochromic film Phys. Med. Biol. 43 2407-12

Constanitinou C, Attix F and Paliwal B 1982 A solid water phantom material for radiotherapy X-ray and gamma ray beam ray calculations Med. Phys. 9 436-41

McLaughlin W 1996 Irradiation of Polymers Fundamentals and Technological Applications (ACS Symp. Ser.) (New York: American Chemical Society) chapter 11

Meigooni A, Sanders M, Ibbott G and Szeglin S 1996 Dosimetric characteristics of an improved radiochromic film Med. Phys. 23 1883-8

Murphy M K, Kovács A, Miller S D and McLaughlin W L 2003 Dose response and post-irradiation characteristics of the Sunna 535-nm photo-fluorescent film dosimeter Radiat. Phys. Chem. 68 981-94

Reinstein L E, Gluckman G R and Meek A G 1998 A rapid colour stabilization technique for radiochromic film dosimetry Phys. Med. Biol. 43 2703-8 